\title{
The actively controlled jet in crossflow
}

\author{
By R. T. M'CLOSKEY ${ }^{1}$, J. M. KING ${ }^{1}$, L. CORTELEZZI ${ }^{2}$ \\ AND A. R. KARAGOZIA ${ }^{1} \dagger$ \\ ${ }^{1}$ Department of Mechanical and Aerospace Engineering, University of California, Los Angeles, \\ CA, 90095-1597, USA \\ ${ }^{2}$ Department of Mechanical Engineering, McGill University, Montreal, Quebec, \\ Canada H3A 2K6
}

(Received 3 June 2001 and in revised form 7 August 2001)

This study quantifies the dynamics of actuation for the temporally forced, round gas jet injected transversely into a crossflow, and incorporates these dynamics in developing a methodology for open loop jet control. A linear model for the dynamics of the forced jet actuation is used to develop a dynamic compensator for the actuator. When the compensator is applied, it allows the jet to be forced in a manner which results in a more precisely prescribed, temporally varying exit velocity, the RMS amplitude of perturbation of which can be made independent of the forcing frequency. Use of the compensator allows straightforward comparisons among different conditions for jet excitation. Clear identification can be made of specific excitation frequencies and characteristic temporal pulse widths which optimize transverse jet penetration and spread through the formation of distinct, deeply penetrating vortex structures.

\section{Introduction}

The canonical flow field associated with the round jet injected normally into crossflow has been extensively studied (Kamotani \& Greber 1972; Fric \& Roshko 1994; Kelso, Lim \& Perry 1996; Broadwell \& Breidenthal 1984; Smith \& Mungal 1998) because of its widespread applications, particularly in air-breathing engines. The vast majority of transverse jet studies have focused on the steady jet, yet active control of the mixing processes associated with the jet in crossflow often requires temporal excitation of the jet flow field. Recent experiments on pulsed transverse jets (Vermeulen, Grabinski \& Ramesh 1992; Kelso et al. 1996; Johari, Pacheco-Tougas \& Hermanson 1999; Schuller et al. 1999; Eroglu \& Breidenthal 2001) suggest that temporally varying the jet velocity $U_{j}$ allows jet penetration and spread to be enhanced at specific conditions of excitation, probably due to control of the temporal generation of jet vorticity. For example, in the study of fully modulated liquid jets in crossflow by Johari et al. (1999), the maximum jet penetration occurs at a forcing frequency, $f$, which corresponds to a jet Strouhal number $S t_{j} \equiv f D / \bar{U}_{j}=0.004$, where $D$ is the inner diameter of the jet orifice and $\bar{U}_{j}$ is the mean jet velocity. Such maxima generally occur for low duty cycles, on the order $\alpha=20 \%$, where the duty cycle $\alpha$ is defined as the temporal pulse width $\tau$ during a given cycle divided by the period $T$ during square wave excitation, taken as a percentage. These results apply for a mean jet-to-crossflow velocity ratio $R \equiv \bar{U}_{j} / U_{\infty}$ of about 5 . In gas-phase experiments by Vermeulen et al. (1992) for an acoustically excited cold jet in hot crossflow, on the

$\dagger$ Author to whom correspondence should be addressed. 
other hand, 'optimal' jet penetration and mixing are achieved for sinusoidal excitation at a jet Strouhal number $S t_{j}=0.22$ and a mean jet-to-crossflow momentum flux ratio $J \equiv \rho_{j} \bar{U}_{j}^{2} / \rho_{\infty} U_{\infty}^{2}=3.13$. All of these 'optimal forcing' Strouhal frequencies are lower than the typical natural or preferred frequencies associated with near-field vortex rollup for the unforced jet in crossflow (Kelso et al. 1996; Schuller et al. 1999). Yet as noted by Cortelezzi \& Karagozian (2001), an appropriately scaled 'preferred' Strouhal frequency for the unforced jet in crossflow should actually account for both jet and crossflow velocities, in addition to jet diameter and other relevant length scales such as the upstream wall boundary layer thickness. Moreover, it is clear from the limited forced transverse jet data available that the optimal forcing conditions, yielding a maximization of transverse jet penetration and spread, may well be configuration- or apparatus-dependent.

In both gas and liquid pulsed transverse jet experiments, a function or signal generator is typically used to produce a temporally varying jet velocity. Experimental evidence (Johari et al. 1999; Schuller et al. 1999) suggests that the temporal variation of the velocity $U_{j}(t)$ at the orifice can be quite different from the input waveform from the signal generator. This difference also appears to be apparatus-dependent. Although the dominant frequency of excitation is generally retained by the jet, differences in the actual waveform shape, duty cycle, and phase can arise from the unsteady flow evolution within the jet plenum-nozzle and/or the jet's nonlinear coupling to the crossflow in the vicinity of the jet orifice. Hence it becomes difficult not only to identify universal scaled excitation conditions (e.g. $\alpha$ and $S t_{j}$ ) that lead to optimal transverse jet penetration and spread, but it is also difficult even to compare results from one experimental configuration to another and from an experiment to a numerical simulation.

The aims of the present study are to understand and quantify the dynamics of the temporally forced jet in crossflow, in particular the dynamics of jet actuation, and to use this information as a means of more precisely controlling jet behaviour. These dynamics are characterized in experiments involving a gaseous round jet injected normally into a uniform gaseous crossflow, but the procedure outlined could apply to other experimental configurations or even to a numerical simulation. A simple linear model for the dynamics of the forced jet allows the development of a dynamic compensator which, when applied, permits the jet to be forced in a manner that results in a more precisely prescribed, temporally varying exit velocity. More importantly, the compensator allows the jet response at the nozzle exit to become essentially independent of the specific characteristics of the jet actuation apparatus, so that a more accurate identification of optimal forcing conditions may be made.

\section{Experimental setup and baseline jet response}

The acoustically pulsed, transverse gas jet is studied here in a small-scale, blowdown wind tunnel. A compressor is used to drive air through the tunnel at speeds that range from 0.5 to $20 \mathrm{~m} \mathrm{~s}^{-1}$ in the test section. The turbulence levels in the tunnel are quantified using a hot-wire anemometry system (Dantec StreamLine with a 55P15 probe) to be less than $3 \%$. The tunnel test section of $12 \mathrm{~cm} \times 12 \mathrm{~cm}$ consists of four sets of ceramic walls (each of length $26 \mathrm{~cm}$ ) which can be replaced by quartz or Plexiglas windows for optical access. In the present studies, the windows are placed so that only near-field visualization of the jet is attained.

The jet nozzle, situated $9.51 \mathrm{~cm}$ downstream of the end of the tunnel contraction, has an inner diameter of $0.75 \mathrm{~cm}$, with a $16: 1$ (area) contraction ratio and a length 

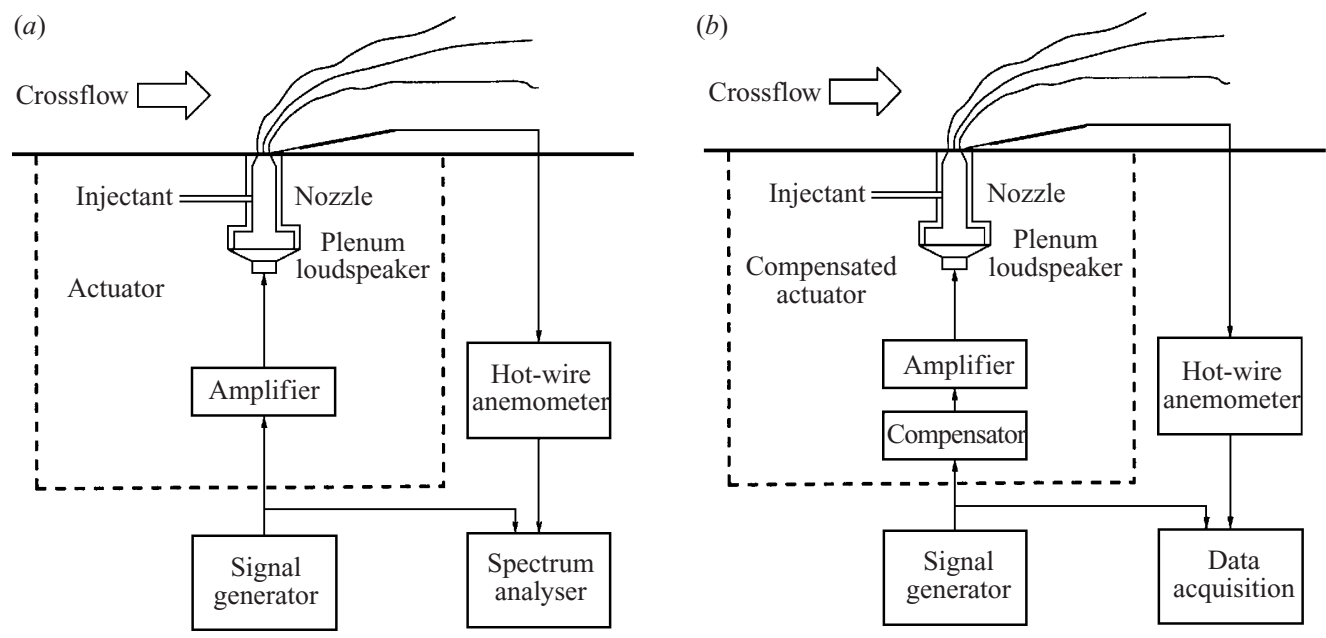

FiguRE 1. Schematic diagrams of the jet actuation system used for identification of jet dynamics (a) and for compensation of jet behaviour $(b)$. A hot-wire anemometry system is used to measure temporal jet response at the exit plane of the jet nozzle. The 'actuator' consists of the jet nozzle, plenum, loudspeaker, and amplifier; the 'compensator' alters the amplifier input to control temporal jet behaviour at the nozzle exit plane, as described in $\S 3$.

of $14 \mathrm{~cm}$. The nozzle is connected to a small plenum with an approximate volume of $150 \mathrm{~cm}^{3}$, sketched as part of the actuator shown in figure 1. The jet fluid (nitrogen) may be introduced in a steady mode or it may be temporally pulsed in an oscillatory fashion by a loudspeaker (Radio Shack $9.16 \mathrm{~cm}$ woofer) which is placed at the bottom of the plenum. A Tylan mass flowmeter is used to measure and verify the mean bulk velocity in the jet during both steady and unsteady operations. The hotwire anemometry system is used to quantify the spatial and temporal velocity profiles at the exit plane of the jet. In all results shown here, the mean jet velocity $\bar{U}_{j}$ is maintained at $3.1 \mathrm{~m} \mathrm{~s}^{-1}$ and the mean crossflow velocity $U_{\infty}$ is $1.2 \mathrm{~m} \mathrm{~s}^{-1}$, for a value of the jet-to-crossflow velocity ratio of $R=2.58$ and a mean jet Reynolds numbers of the order 1500 . A virtually flat spatial distribution in velocity is quantified at the jet exit plane during steady jet operation.

Jet visualization in the present experiments is achieved via smoke generation upstream of the jet nozzle/plenum. A heated seeder with a liquid parafin solution is used for this purpose; nitrogen passing through the seeder then travels through an ice bath to create the desired smoke concentration at temperatures equal to that of the crossflow air. A $500 \mathrm{~W}$ white light is fixed above the test section to illuminate the jet, while the ceramic test section walls are sooted to improve resolution. A Nikon N2000 SLR camera is used to obtain images of the evolution of smoke in the acoustically pulsed jet. When the jet is acoustically forced, the loudspeaker is driven by an amplifier that accepts input from a signal generator (Keithly Model 3940 Multifunction Synthesizer) indicated in figure 1. The operation of the compensated actuator shown in figure $1(b)$ will be described in $\S 3$.

Sample results are shown in figure $2(a-d)$ for the input waveform delivered to the amplifier and loudspeaker from the signal generator, and the response measured by the hot-wire anemometer at the centre of the jet exit plane. Mean values are subtracted from the input as well as output signals (in volts). The input waveform is recovered reasonably well in the velocity at the jet exit for sinusoidal jet excitation. For square wave excitation, however, the shape of the input signal is not accurately 

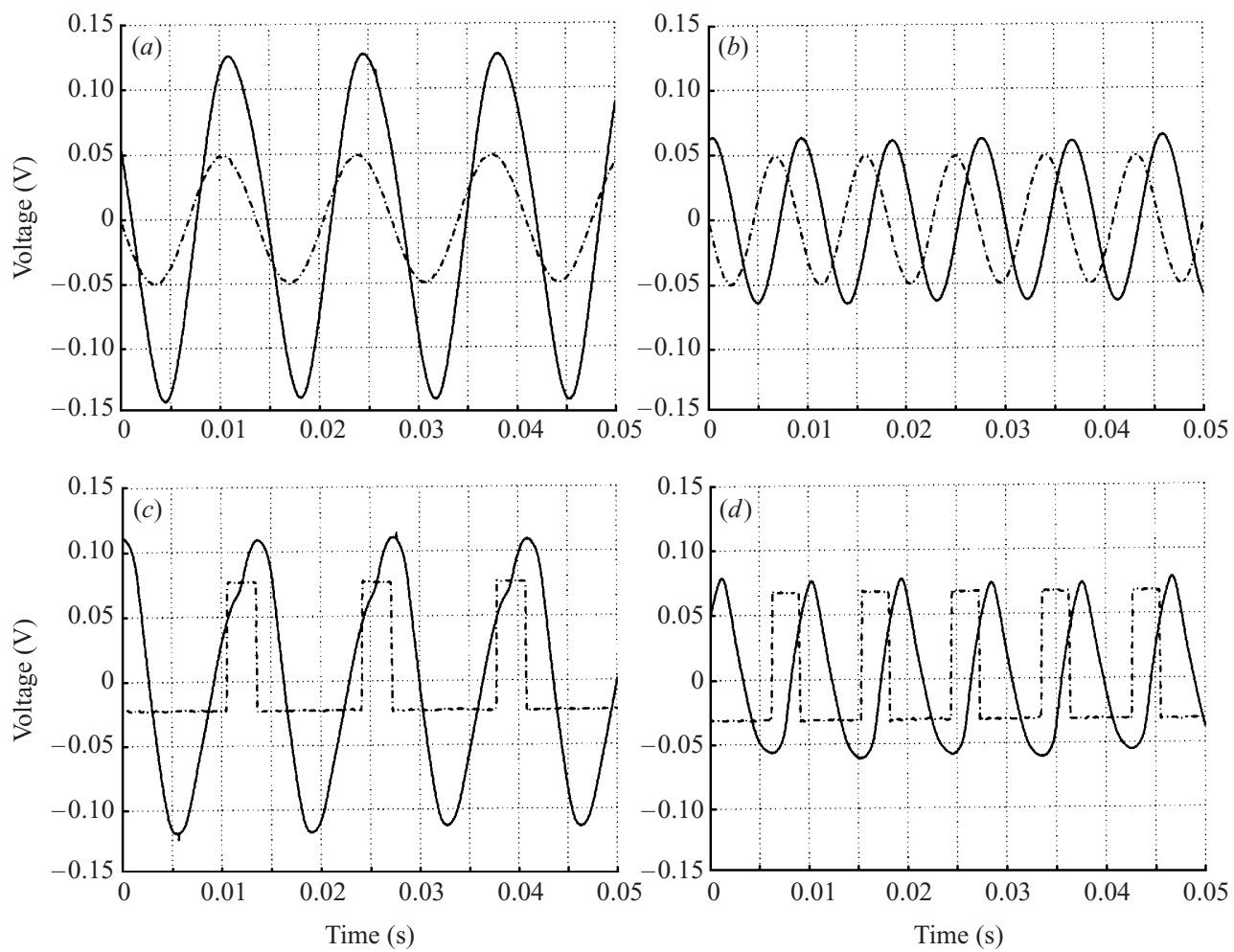

FIGURE 2. Experimental 'input' voltage delivered by the signal generator and amplifier (dash-dotted line), with the corresponding mean-subtracted voltage output measured at the centerline of the jet exit plane by the hot-wire anemometer (solid line). There is no control or compensation here. Results are shown for $(a)$ sine wave input at $73.5 \mathrm{~Hz},(b)$ sine wave input at $110 \mathrm{~Hz},(c)$ square wave input at $73.5 \mathrm{~Hz}$ with $\alpha=22 \%$, and $(d)$ square wave input at $110 \mathrm{~Hz}$ with $\alpha=31 \%$. The input gain to the amplifier is the same in $(a)$ and $(b)$ and in $(c)$ and $(d)$, and $R \equiv \bar{U}_{j} / U_{\infty}$ is maintained at 2.58 for all cases.

recovered at the jet exit plane, but rather resembles a somewhat peaked, non-uniform sine wave. As a consequence, the actual jet excitation has an effective duty cycle that differs significantly from that of the input signal; this phenomenon was also observed by Johari et al. (1999) in liquids. Moreover, the amplitude of the response of the jet is dependent on the frequency of excitation, as seen by comparing the jet output amplitudes between figures $2(a)$ and $2(b)$ and between figures $2(c)$ and $2(d)$.

This frequency response of the jet/actuator may be characterized more extensively using a spectrum analyser (HP Model 3856) as shown in figure 1(a). When the signal generator is made to produce a broad-band amplifier input signal, the spectrum analyser, processing both this excitation signal and the hot-wire response, can take the ratio of the two signals to estimate the frequency response. The frequency response between 40 and $1640 \mathrm{~Hz}$ is shown by the thick dashed lines in figure 3 . The two plots represent the amplitude $(a)$ and phase $(b)$ of the hot-wire voltage output relative to the input signal. In this frequency range, the hot-wire anemometer dynamics have almost no phase lag or amplitude variation, according to the manufacturer, and thus the hot-wire output voltage is a representation of the jet velocity via King's Law.

The Bode plots in figure 3 reveal that there is a maximum amplitude of the jet response at an excitation frequency of approximately $73.5 \mathrm{~Hz}$, and that the response 

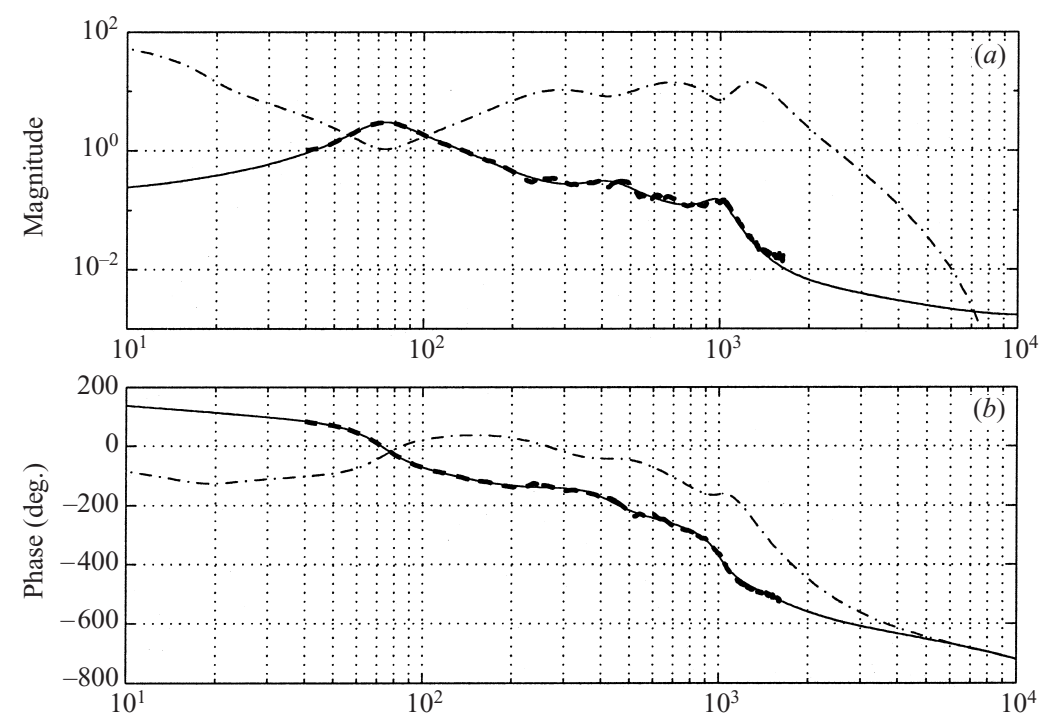

$(\mathrm{Hz})$

FiguRE 3. Bode diagrams describing the experimental frequency response of the jet/actuator at the nozzle exit (thick dashed line); these represent the magnitude and phase of the ratio of the output (hot wire) to input (function generator) signals. Also shown is the frequency response of an eight-state linear model representing the actuator dynamics mathematically (solid lines). The compensator frequency response (thin dash-dotted lines) represents an inversion of the model which is applied in the experiment to create a more uniform frequency response.

of the jet rolls off substantially at frequencies above $100 \mathrm{~Hz}$. The peak amplitude at $73.5 \mathrm{~Hz}$ occurs at about one-third the value of the near-field vortex shedding frequency (approximately $220 \mathrm{~Hz}$ ) for the unforced jet in crossflow at the present values of $\bar{U}_{j}$ and $U_{\infty}$. This near-field vortex shedding frequency is quantified in the absence of any acoustic excitation from hot-wire measurements (taken at both the centre of the jet exit and in the upstream shear layer) as well as from smoke images. The non-uniform frequency response shown by the thick dashed lines in figure 3 is undesirable; a frequency response with flat magnitude and linear phase lag over a broad frequency band is required in order to be able to reproduce the desired square waveform excitation at the jet exit.

\section{Linear system model and compensator}

A linear model representing the dynamics of the nozzle/plenum/speaker/amplifier (herein referred to as the actuator) may be developed based on these experimental measurements of jet response. This mathematical model becomes an integral component in developing a control strategy for improving the jet response.

The mathematical model of the actuator dynamics consists here of an eight-pole transfer function. Parameters in this transfer function are obtained via an off-line fit to the experimental frequency response data using the Sanathanan \& Koerner (1963) algorithm. The frequency response of the resulting model is shown by the solid line in figure 3. The model appears to replicate the actuator response very well in the frequency range of interest.

This actuator model is then used to synthesize a discrete-time reference-signalshaping filter. The objective here is to design this shaping filter such that the series 



$(\mathrm{Hz})$

FIGURE 4. Bode plots representing the frequency response of the compensated jet on a linear frequency scale. The solid line shows the compensated actuator, while the uncompensated actuator is shown (for comparison) by the dashed line.

connection of the filter and the actuator yields a flat frequency response magnitude with a linear phase lag response. This type of frequency response should improve reproduction of the input signal at the jet exit plane, especially for square wave excitation, with only a time delay between input and output. The shaping filter is designed by solving a weighted $\mathscr{H}_{\infty}$ model matching problem using the Matlab Mutools Toolbox (see Balas et al. 1995 for details). This type of model matching problem usually involves the mathematical inversion of the identified model. The inversion can lead to large filter gains, however, and hence an undesirable sensitivity to high-frequency noise. Thus a fourth-order Bessel filter with an $800 \mathrm{~Hz}$ corner frequency is applied to the shaping filter output in order to attenuate the gain beyond $800 \mathrm{~Hz}$. A Bessel filter is chosen to preserve the desired linear phase response.

The series connection of the shaping filter and the Bessel filter are collectively denoted the compensator for the jet actuation, shown schematically by the block in figure $1(b)$. The frequency response of the compensator itself is shown by the thin dash-dotted lines in figure 3, representing the applied excitation to the amplifier required to produce the desired frequency response (i.e. flat in terms of magnitude and linear in terms of phase). Note that the magnitude plot of the compensator in figure 3 is essentially an inverse of the actuator model magnitude. In these experiments, the shaping filter is implemented on a commercial digital signal processor (dSpace model 1103 DSP), while the Bessel filter remains analog.

Application of the compensator to the jet data results in the compensated jet frequency response shown by the solid lines in figure 4 . For reference, the uncompensated jet response is shown by the dashed lines; this is the same quantity as is represented by the thick dashed lines in figure 3. A linear frequency scale is used in figure 4 to further distinguish the difference between the compensated and uncompensated cases. The desired linear phase lag characteristic and almost constant magnitude in frequency response are both achieved in the compensated system. It should be noted 

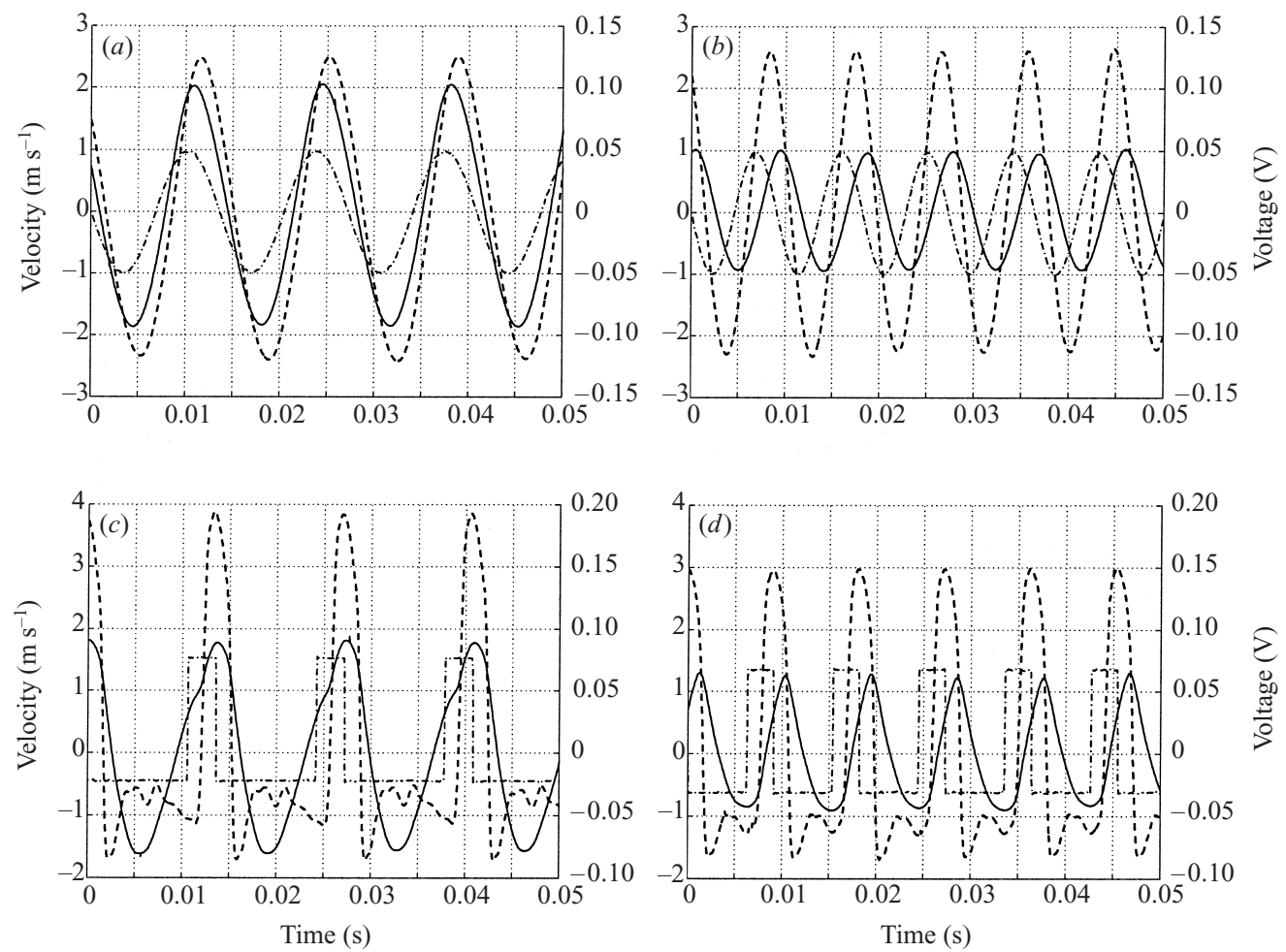

FiguRE 5. Comparison of the input waveform to the actuator (dash-dotted line), in volts, with the measured velocity perturbations (i.e. with the mean subtracted) at the jet exit, in $\mathrm{m} \mathrm{s}^{-1}$. Results shown are for velocity perturbations without compensation (solid lines) and with compensation (dashed lines). The compensated results correspond to the cases in which $U_{j, R M S}^{\prime}$, the RMS of the velocity perturbations, is matched among all excitation conditions $(a-d)$ at a value of $1.7 \mathrm{~m} \mathrm{~s}^{-1}$. The uncompensated velocity perturbations correspond to the mean-subtracted voltage output waveforms shown in figure 2. Here $\bar{U}_{j}=3.1 \mathrm{~m} \mathrm{~s}^{-1}$ and $U_{\infty}=1.2 \mathrm{~m} \mathrm{~s}^{-1}$, with $(a)$ sine wave input at $73.5 \mathrm{~Hz},(b)$ sine wave input at $110 \mathrm{~Hz},(c)$ square wave input at $73.5 \mathrm{~Hz}$ with $\alpha=22 \%$, and $(d)$ square wave input at $110 \mathrm{~Hz}$ with $\alpha=31 \%$.

that the RMS amplitude of the velocity perturbations, $U_{j, R M S}^{\prime}$, may be matched for the compensated jet among different excitation conditions by adjusting the amplifier gain and applying King's Law to convert voltage to velocity. When $U_{j, R M S}^{\prime}$ is matched among different excitation conditions, the real effect of applied frequency and duty cycle on jet behaviour may be ascertained.

The effect of the compensator on the actual waveform output at the jet exit (with the mean subtracted) is shown by the examples in figure 5. Results are shown for the uncompensated jet response (in $\mathrm{m} \mathrm{s}^{-1}$ ) and for a compensated jet in which the RMS amplitude of the velocity perturbations, $U_{j, R M S}^{\prime}$, is matched among different excitation conditions at a value of $1.7 \mathrm{~m} \mathrm{~s}^{-1}$. The compensator is seen to have relatively little effect on the actual sine waveform, other than altering the amplitude of the jet response to become essentially independent of frequency. The compensator has a significant effect on square waveform excitation, however, not only in altering the amplitude of excitation, but also in forcing the waveform to more closely resemble a square wave with essentially the desired duty cycle. The relatively low-level undershoot observed in the compensated square wave signal is due to nonlinearities in the actuator dynamics 
which are not represented by the model. As will be seen, the overall improvement in square waveform shape by the compensator has a significant effect on jet behaviour.

\section{Results}

Smoke visualization now reveals the effects of compensation and of different excitation conditions on jet behaviour. In the present set of experiments, the gain to the amplifier is adjusted so that for all excitation frequencies and duty cycles explored, including both uncompensated and compensated jets, $U_{j, R M S}^{\prime}$ is fixed at approximately $1.7 \mathrm{~m} \mathrm{~s}^{-1}$.

As a baseline reference, smoke visualization for the unforced jet in crossflow with a jet-to-crossflow velocity ratio of $R=2.58$ is shown in figure $6(a)$. Effects of the actuator compensation during random noise excitation show that both the uncompensated and compensated jet look nearly identical to the unforced jet. Since acoustic energy is supplied to the jet at a wide range of random frequencies, the jet is unable to respond to any one frequency or group of frequencies in a coherent way. These observations suggest that random excitation is inefficient for mixing enhancement.

When sine wave excitation is applied to the jet, compensation has the primary benefit of equalizing the magnitude of jet response at higher frequencies. Figures $6(b)$ and $6(c)$ display jet evolution for uncompensated and compensated jets, respectively, perturbed by sinusoidal excitation at $73.5 \mathrm{~Hz}$. The effect of the relatively minor change in waveform (see figure $5 a$ ) on jet evolution is rather small, and since the waveform is nearly the same, compensation has little effect. For most other sine wave excitation cases, the compensated jet looks similar to that at $73.5 \mathrm{~Hz}$. Sine wave excitation is thus seen to have relatively little impact on jet penetration and spread compared with the unforced jet.

Jet compensation makes a significant difference when square wave excitation of the jet is desired, especially at specific frequencies and specific values of the pulse width $\tau$. Figures $6(d)$ and 6(e) show smoke images for jet evolution without and with compensation, respectively, for square wave excitation at $110 \mathrm{~Hz}$ and a duty cycle of $31 \%$. As indicated in figure $5(d)$, the primary difference between uncompensated and compensated jet response at the nozzle exit for this frequency is in the waveform shape. Figures $6(d)$ and $6(e)$ demonstrate that, with compensation of the square wave, deeply penetrating and rather distinct vortex rings are introduced into the flow field at the frequency of excitation, resulting in significant increases in jet penetration and spread. It is clear from hot-wire measurements that these results are largely achieved because of the ability of the compensated jet to more accurately replicate the distinct pulsations of fluid introduced by a square wave.

Jet compensation permits a comparison of the effects of excitation frequency and duty cycle on jet behaviour, since $U_{j}^{\prime}$ has been made independent of frequency. The improved jet penetration and spread during square wave excitation is actually seen to occur only for select frequencies and pulse widths $\tau$. Similar to the jet behaviour in figure $6(e)$, when square wave excitation is applied at $55 \mathrm{~Hz}$ and $15 \%$ duty cycle (figure $6 f$ ) and at $73.5 \mathrm{~Hz}$ and $22 \%$ duty cycle (figure $6 \mathrm{~g}$ ), individual vortex rings are formed which penetrate well into the crossflow. These three cases all have approximately the same input pulse width $\tau$, within the range $2.7-3.0 \mathrm{~ms}$. When the duty cycle (and hence $\tau$ ) is increased so that $\tau>3 \mathrm{~ms}$ for a given frequency, the penetration and spread of the jet are significantly reduced. When the duty cycle is reduced so that $\tau<2.7 \mathrm{~ms}$, reasonably good penetration and spread are still achieved, 
(a)

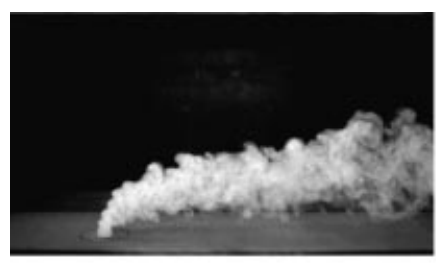

(d)



(g)

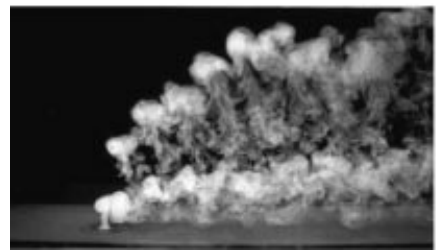

(b)

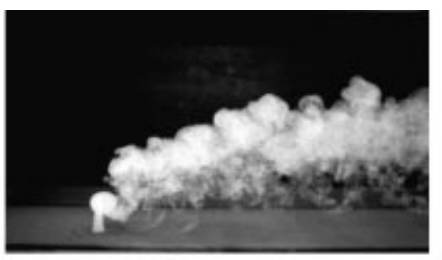

(e)

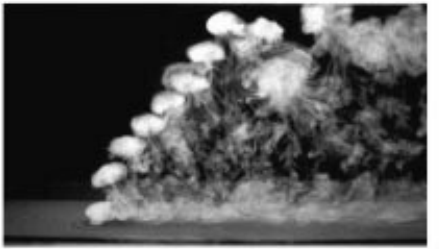

(h)

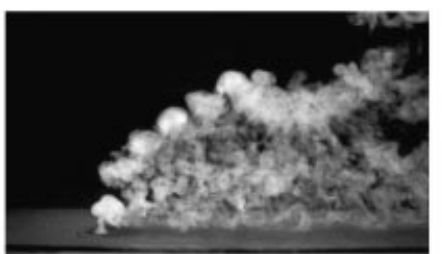

$(c)$

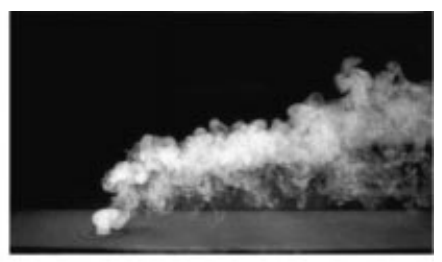

$(f)$

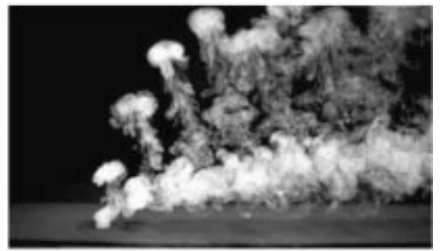

(i)

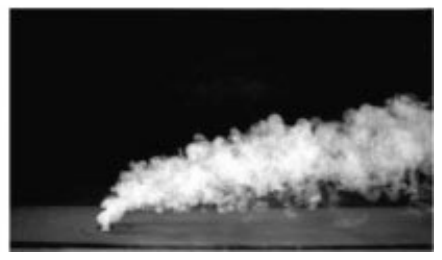

FIGURE 6. Smoke visualization of the jet in crossflow at an average jet-to-crossflow velocity ratio of $R=2.58$, where $\bar{U}_{j}=3.1 \mathrm{~m} \mathrm{~s}^{-1}$ and $\bar{U}_{\infty}=1.2 \mathrm{~m} \mathrm{~s}^{-1}$, and for an RMS amplitude of the velocity perturbations, $U_{j}^{\prime}$, matched at $1.7 \mathrm{~m} \mathrm{~s}^{-1}$. Images shown are for photographs taken with an exposure time of $1 / 2000 \mathrm{~s}$, yielding an 'instantaneous' image. Results shown are for: $(a)$ the 'unforced' jet (in the absence of any acoustic excitation), (b) 'uncompensated' sine wave excitation at $73.5 \mathrm{~Hz}$, (c) 'compensated' sine wave excitation at $73.5 \mathrm{~Hz},(d)$ 'uncompensated' square wave excitation at $110 \mathrm{~Hz}$ and a duty cycle of $31 \%,(e)$ 'compensated' square wave excitation at $110 \mathrm{~Hz}$ and a duty cycle of $31 \%,(f)$ 'compensated' square wave excitation at $55 \mathrm{~Hz}$ and a duty cycle of $15 \%,(g)$ 'compensated' square wave excitation at $73.5 \mathrm{~Hz}$ and a duty cycle of $22 \%,(h)$ 'compensated' square wave excitation at $85 \mathrm{~Hz}$ and a duty cycle of $24 \%$. (i) 'compensated' square wave excitation at $220 \mathrm{~Hz}$ and a duty cycle of $62 \%$.

consistent with the observations of Johari et al. (1999), yet they are not as great nor as visibly evident as in figure 6(e-g).

The observation of an optimal temporal pulse width $\tau$ to achieve deeply penetrating pulses of vorticity also appears to be consistent with the suggestion by Gharib, Rambod \& Shariff (1998) of the existence of a universal time scale (or 'time of pinchoff') required for coherent vortex ring formation. These researchers find that a piston-generated vortex ring attains a maximum circulation and is disconnected from its trailing jet at critical values of the ratio of piston stroke to diameter, corresponding to a 'universal time scale of vortex formation'. While the present experiments involve the acoustically pulsed, continuous jet, rather than piston-driven starting vortex rings, the observation of a critical time scale for distinct ring formation (as seen in figure $6 e-$ $g$ ) suggests a similar physical mechanism for enhanced penetration.

The frequencies of square wave excitation in figures $6(e), 6(f)$, and $6(\mathrm{~g})(110 \mathrm{~Hz}$, $55 \mathrm{~Hz}$, and $73.5 \mathrm{~Hz}$, respectively) generate the highest observed level of jet penetration. These cases represent frequencies which are, respectively, one-half, one-quarter, and one-third the preferred frequency for the unforced jet in crossflow under these flow conditions (approximately $220 \mathrm{~Hz}$ ). Square wave excitation at $44 \mathrm{~Hz}$ (one-fifth of 
$220 \mathrm{~Hz}$ ) and a duty cycle of $13 \%$ (corresponding to $\tau=2.95 \mathrm{~ms}$ ) yields similar results to those in figure $6(e-g)$. Hence when distinct pulses of fluid are introduced at periods which are two, three, four, or five times that associated with natural vortex shedding for the jet, and with a specific pulse width in the range $\tau \approx 2.7-3.0 \mathrm{~ms}$, significant jet penetration can be achieved. When compensated jet excitation is applied at non-subharmonic frequencies but with duty cycles corresponding to $\tau \approx 2.7-3.0 \mathrm{~ms}$, jet penetration and spread are not as extensive as at the subharmonic frequencies. This is indicated, for example, in figure $6(h)$ for $85 \mathrm{~Hz}$ excitation with a duty cycle, $24 \%$, corresponding to $\tau=2.82 \mathrm{~ms}$. Compensated square wave excitation at the preferred frequency for the unforced jet, $220 \mathrm{~Hz}$, with a duty cycle corresponding to $\tau=2.82 \mathrm{~ms}$, produces jet penetration which is only slightly improved over the unforced jet, as indicated in figure 6(i). In fact, in no instance did square wave excitation at frequencies higher than about $140 \mathrm{~Hz}$ yield good jet penetration. It appears that when the duty cycle $\alpha$ must be close to or greater than $50 \%$ in order to achieve pulse widths of $\tau \approx 3 \mathrm{~ms}$ for a specified frequency, distinct pulses of vorticity and hence strong jet penetration are difficult to achieve.

\section{Conclusions}

Characterization of the dynamics of the actuation system for the forced jet in crossflow is described here, and a robust methodology is evolved whereby these dynamics may be used to develop a jet compensation system for open loop jet control. Application of the compensator allows significantly improved temporal waveforms to be attained at the jet exit, so that accurate comparisons among different conditions of jet excitation can be made. Optimal jet penetration and spread are observed to occur for square wave excitation at subharmonics of the 'natural' vortex shedding frequency for the jet (at one-fifth, one-quarter, one-third, and one-half of this shedding frequency). In all optimal cases, however, maximum jet penetration and spread correspond to square wave excitation at duty cycles corresponding to a specific value of excitation pulse width $\tau$ of approximately $2.7-3.0 \mathrm{~ms}$. At other duty cycles (and at other frequencies), jet penetration is not as great. This characteristic value of $\tau$ appears to depend on specific experimental conditions (e.g. jet velocity, crossflow velocity, etc.), and merits further exploration. The present approach defines a methodology by which this dependence, and other aspects of optimizing the forced jet in crossflow, may be studied with confidence.

The authors wish to acknowledge the assistance of Thierry Schuller of Ecole Centrale de Paris, Robert Lobbia and Ari Majamaki of UCLA, and Yungmo Kang of Capstone Turbine Corp. in the early stages of this work. This research has been supported by the Energy Innovations Small Grants Program of the California Energy Commission under grant 51240A/99-01-56, by NASA Dryden Flight Research Center under Grant NCC 2-374, by NSERC Grant RGPIN217169, by NSF grant ECS-9985046, and by the University of California Energy Institute.

\section{REFERENCES}

Balas, G. J., Doyle, J. C., Glover, K., Packard, A. \& Smith, R. $1995 \mu$-Analysis and Synthesis Toolbox. The MathWorks, Natick, MA.

Broadwell, J. E. \& Breidenthal, R. E. 1984 Structure and mixing of a transverse jet in incompressible flow. J. Fluid Mech. 148, 405-412. 
Cortelezzi, L. \& Karagozian, A. R. 2001 On the formation of the counter-rotating vortex pair in transverse jets. J. Fluid Mech. 446, 347-373.

Fric, T. F. \& Roshko, A. 1994 Vortical structure in the wake of a transverse jet. J. Fluid Mech. 279, $1-47$.

Eroglu, A. \& Breidenthal, R. E. 2001 Structure, penetration, and mixing of pulsed jets in crossflow. AIAA J. 39, 417-423.

Gharib, M., Rambod, E. \& Shariff, K. 1998 A universal time scale for vortex ring formation. J. Fluid Mech. 360, 121-140.

Johari, H., Pacheco-Tougas, M. \& Hermanson, J. C. 1999 Penetration and mixing of fully modulated turbulent jets in crossflow. AIAA J. 37, 842-850.

Kamotani, Y. \& Greber, I. 1972 Experiments on a turbulent jet in a cross flow. AIAA J. 10, $1425-1429$.

Kelso, R. M., Lim, T. T. \& Perry, A. E. 1996 An experimental study of round jets in cross-flow. J. Fluid Mech. 306, 111-144.

Sanathanan, C. K. \& Koerner, J. 1963 Transfer function synthesis as a ratio of two complex polynomials. IEEE Trans. Automatic Control 8, 56-58.

Schuller, T., King, J., Majamaki, A., Karagozian, A. R. \& Cortelezzi, L. 1999 An experimental study of acoustically controlled gas jets in crossflow. Bull. Am. Phys. Soc. 44(8), 111.

Smith, S. H. \& Mungal, M. G. 1998 Mixing, structure and scaling of the jet in crossflow. J. Fluid Mech. 357, 83-122.

Vermeulen, P. J., Grabinski, P. \& Ramesh, V. 1992 Mixing of an acoustically excited air jet with a confined hot crossflow. ASME Trans: J. Engng Gas Turb. Power 114, 46-54. 